\title{
A Comparison of Radiographic Joint Space Width Measurements Versus Ultrasonographic Assessment of Cartilage Thickness in Children with Juvenile Idiopathic Arthritis
}

\author{
Dan Østergaard Pradsgaard (D, Arne Hørlyck, Anne Helene Spannow, Carsten Heuck, \\ and Troels Herlin (i)
}

ABSTRACT. Objective. Joint space narrowing (JSN) is a measurable outcome of tissue degeneration in arthritis. JSN is usually assessed by conventional radiography. Ultrasonographic (US) measurement of joint cartilage thickness has been validated in healthy children, and US measurement of the distal femoral cartilage has been validated in a group of patients with juvenile idiopathic arthritis (JIA). Our aim was to compare the measures of cartilage thickness of the proximal cartilage site in the second metacarpophalangeal (MCP), second proximal interphalangeal (PIP), and knee joints as assessed by US to joint space width (JSW) as measured by computerized radiography in children with JIA.

Methods. The study included 74 children with JIA aged 5-15 years (median 11.3 yrs). MCP and PIP joints were assessed at one midline spot. Knee joints were assessed at the medial and lateral femoral condylar areas. Only the proximal cartilage site in the joints was assessed by US, whereas the complete JSW was assessed by radiography.

Results. We assessed 136 second MCP, 138 second PIP, and 146 knee joints. We found a high level of agreement between US and radiographic measures of cartilage thickness and JSW: $r=0.82-0.86$ (second MCP), $\mathrm{r}=0.50-0.55$ (second PIP), and $\mathrm{r}=0.52-0.81$ (knee); $\mathrm{p}<0.001$ for all 8 assessed sites.

Conclusion. US measurements of cartilage thickness of the proximal site of the second MCP, second PIP, and knee joints correlated well with radiographic JSW measurements in the finger and knee joints of children with JIA. However, US does not measure the distal cartilage, which may limit its use in the assessment of JSN. (First Release November 15 2018; J Rheumatol 2019;46:301-8; doi:10.3899/ jrheum.170571)

Key Indexing Terms:

ULTRASOUND

CARTILAGE

RADIOLOGY

JUVENILE IDIOPATHIC ARTHRITIS JOINT SPACE WIDTH

Juvenile idiopathic arthritis (JIA) is the most common rheumatic disease in childhood, and it comprises a heterogeneous group of diseases that cause synovitis and joint destruction. The functional disability experienced in JIA is caused primarily by the degeneration of the osteocartilaginous structures owing to the inflammatory process in the

From the Department of Pediatrics, and the Department of Radiology, Aarhus University Hospital, Aarhus, Denmark.

Supported by the Danish Rheumatism Association and by the Aarhus University Research Foundation.

D.Ø. Pradsgaard, MD, PhD, Department of Pediatrics, Aarhus University Hospital; A. Hørlyck, MD, Department of Radiology, Aarhus University Hospital; A.H. Spannow, MD, PhD, Department of Pediatrics, Aarhus University Hospital; C. Heuck, MD, PhD, Department of Pediatrics, Aarhus University Hospital; T. Herlin, MD, DMSc, Department of Pediatrics, Aarhus University Hospital.

Address correspondence to Dr. D.Ø. Pradsgaard, Aarhus University Hospital Skejby, Pediatrics, Palle Juul-Jensens Boulevard 99, Aarhus N 8200, Denmark.E-mail: dan.pradsgaard@rm.dk

Accepted for publication September 7, 2018. synovium ${ }^{1}$. Joint space narrowing (JSN), which is primarily the result of cartilage thinning, is a measurable outcome of chronic arthritis activity along with epiphyseal overgrowth and juxtaarticular osteopenia ${ }^{2}$. Late destructive changes in addition to the progression of JSN include subchondral bone erosion and finally, ankylosis of the joint. Therefore, early detection of the inflammatory process and of osteocartilaginous degeneration are of great importance for devising an optimal treatment strategy.

The main imaging modalities in pediatric rheumatology are conventional radiography, computed tomography, magnetic resonance imaging (MRI), and within the last decade, ultrasonography (US) ${ }^{3}$. Radiography has often been the initial choice for evaluating structural damage because of its low cost, high-resolution images, and easy access, and it may be helpful in ruling out differential diagnoses to JIA. Regarding early inflammatory changes in the joint, radiography has some disadvantages, because soft tissue compo-

Personal non-commercial use only. The Journal of Rheumatology Copyright (c) 2019. All rights reserved. 
nents have similar radiographic density and therefore cannot be clearly differentiated. For visualizing later outcomes of arthritic diseases, radiography is a cheap, highly available modality for cartilage degradation (including JSN), osteopenia, erosion, ankylosis, and malalignment of the joint $^{4}$. The use of ionizing radiation in radiographs is an ethical problem, although the amount of radiation has decreased with the use of computerized radiography ${ }^{5}$.

US is a reliable modality for assessing joint cartilage thickness in healthy children. In previous studies, our group validated US for the assessment of joint cartilage thickness in healthy children ${ }^{6,7}$, and we found decreased cartilage thickness in the knees of children with JIA after controlling for sex and age ${ }^{8,9}$. We further validated US measurement of cartilage thickness by comparing US measurements to MRI measurements in healthy children and children with JIA ${ }^{6,10}$.

No studies have compared radiographic assessment of joint space width (JSW) with the changes in joint cartilage thickness as assessed by US. The aim of our current study was therefore to correlate measures of cartilage thickness in the knee, second metacarpophalangeal (second MCP), and second proximal interphalangeal (second PIP) joints to the measures of JSW performed by computerized radiography in children with JIA.

\section{MATERIALS AND METHODS}

We invited 95 children (7-15 yrs old) who were diagnosed with JIA according to the 2001 revised International League of Associations for Rheumatology classification ${ }^{11}$ and who were followed at our pediatric rheumatology clinic to participate in the study at least 1 month prior to the examination date. The inclusion criteria were systemic JIA, persistent and extended oligoarticular JIA, and rheumatoid factor (RF)-positive or RF-negative polyarticular JIA. Their parents provided informed consent. Patients were excluded if they had received intraarticular corticosteroid injection (IACI) within 1 month prior to examination or had a history of previous joint surgery. The study was approved by the local ethics committee (M-20070044). The US and clinical examinations were conducted within 1-13 days of radiography. The radiographic investigation was planned after the parents provided informed consent.

Clinical examination. Joint activity was assessed by an experienced pediatric rheumatologist and was defined as swelling within the knee joint or limitation in the range of joint movement with joint pain or tenderness. For each patient with JIA, we established an adapted Juvenile Arthritis Disease Activity Score-10 that consisted of the active joint count of 10 joints (bilateral knee, ankle, wrist, second MCP, and second PIP joints), erythrocyte sedimentation rate, parent's global assessment, and physician's global assessment ${ }^{12}$. Signs of active arthritis were valued dichotomously as $0=$ no active joint and $1=$ active joint.

Medical records. Medical records were reviewed regarding a history of previous joint activity in the knee, second MCP, and second PIP joints and overall disease duration, which was defined as the first sign of arthritic activity that was confirmed by a physician.

US examination. A Hitachi EUB 7500 scanner with a 6-14-MHz linear transducer (EUP-L65) was used for the examination. All examinations were performed by the same observer. The probe pressure was adjusted to a level just below visible deformation on the anatomical structure. The gain, focus, and depth of the US were adjusted to optimize the images and the other settings were the same in all examinations. When assessing cartilage thickness in the knee, each child was in a supine position with the knee joint maximally flexed, and the transducer was held in a cross-sectional position as described previously ${ }^{8}$. Cartilage was measured at the medial and lateral condyles at the point at which a horizontal line hits the cartilage surfaces tangentially; it was also measured and at the midline of the intercondylar notch (Figure 1A).

To assess the finger joints, the joints were flexed at a $90^{\circ}$ angle. The transducer was held longitudinally and orthogonally along the midline of the proximal or intermediate phalanges to assess the second MCP and the second PIP, respectively, resulting in a tangential angle to the cartilage surface (Figure $2 \mathrm{~A})^{8}$.

Radiographic examination. Radiography was performed on both knees in the coronal and sagittal planes with the child in a supine position. JSW was measured orthogonally from the surface of the lateral and medial femoral condyles to the surface of the tibial bone (Figure 1B). We did not measure the distance from the intercondylar notch of the femoral bone to the surface of the tibial bone owing to the recess in the femur that is hidden by the more anterior part of the femoral bone surface on plain radiographs.

Radiographs of the second MCP and second PIP joints were performed with the child's palms resting on the table and the fingers stretched. The JSW of the second MCP was measured in the midline orthogonally between the second metacarpal and proximal phalangeal bones, and the JSW of the second PIP was measured between the proximal and intermediate phalangeal bones (Figure 2B).

Data collection. The examiners who measured the JSW on the computed radiography images and the US images and the clinical investigator were blinded to each other's scores. In addition, the individuals who determined the JSW on the computed radiography and US images were blinded to the clinical information (age, sex, JIA subcategories, disease duration, and treatment).

Statistical analysis. For statistical analysis we used STATA 11 (StataCorp) and IBM SPSS statistics v. 24 software.

Correlation between JSW and US-measured cartilage thickness was analyzed using Spearman's rank and intraclass correlation coefficient. Regression analysis was used to compare active and inactive joints. Significance level was set at $\mathrm{p}<0.05$.

\section{RESULTS}

Of the 95 children with JIA aged 5-15 years who were invited to participate in the study, 90 agreed. Of these 90 children, 1 cancelled because of illness on the day of radiography, 7 declined because we were not able to schedule the radiography on the same date as the other examinations, and 8 never showed up for the scheduled radiographic examination. The study thus included 74 children (54 girls and 20 boys) with a median age of 11.3 years (range 5-15 yrs). Their demographic and anthropometric data are shown in Table 1. The sex and age distribution varied among the JIA subtypes. The JIA cohort included 10 patients with systemic JIA, 4 with RF-positive JIA, 17 with RF-negative polyarticular JIA, and 15 with extended and 28 with persistent oligoarticular JIA. Of the 148 knees, 102 (69\%) had a history of active arthritis; of these, $88(86 \%)$ had received IACI at least once (range 1-11 injections).

Of the 148 knee, second MCP, and second PIP joints that we planned to investigate, US data were missing for 2 left knee joints, for 6 left and 6 right MCP joints, and for 5 left and right PIP joints. Radiographic data were missing for 1 right knee joint and for 1 left and right MCP and 1 PIP joint. Thus, we compared measurements for 72 left and 73 right 

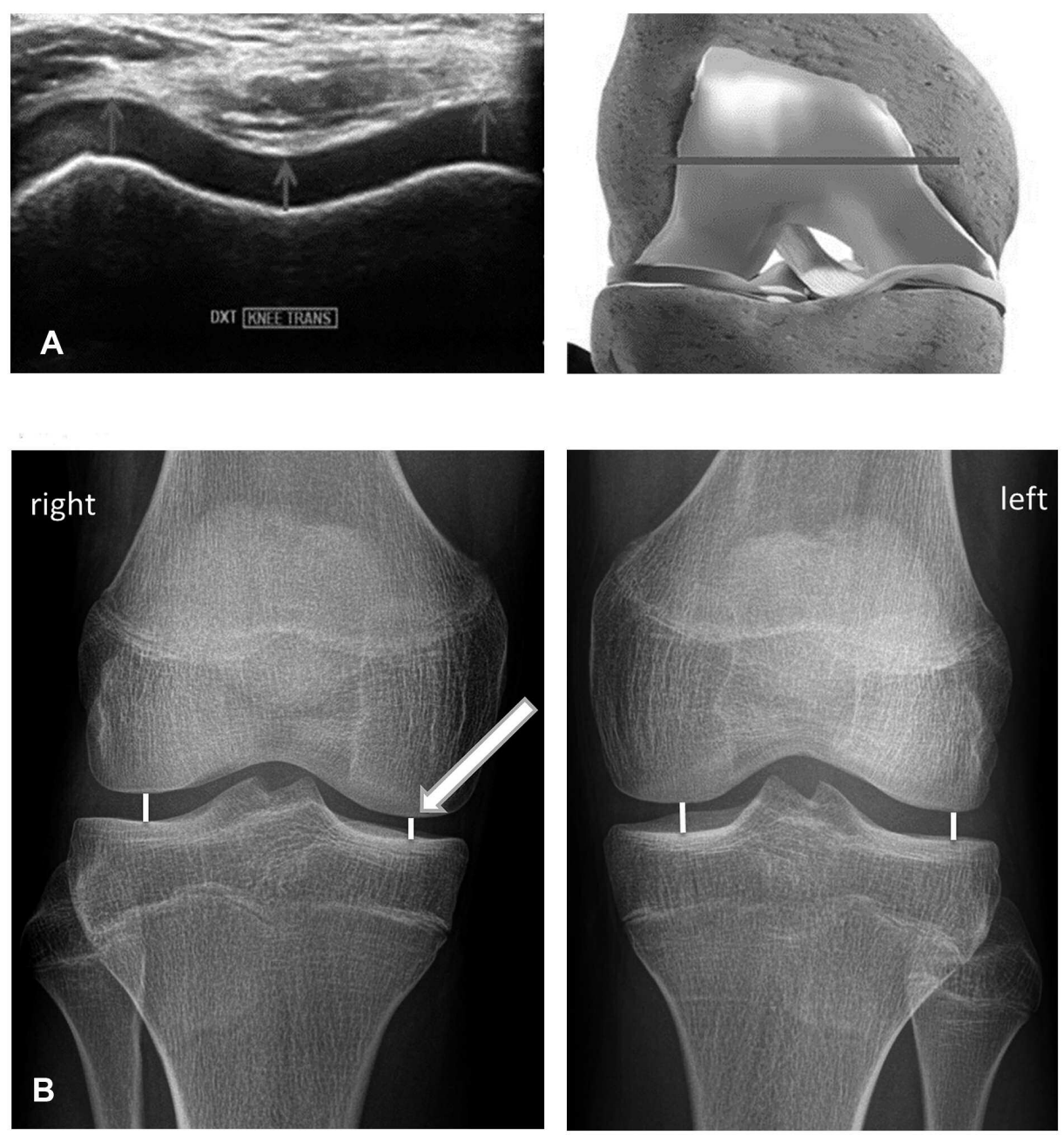

Figure 1. A. Ultrasound assessment of knee cartilage thickness. The grey arrows and line indicate the points of assessment. B. Radiographic assessment of joint space width. White lines indicate points of assessment.

knee joints, for 68 left and right second MCP joints, and for 69 left and right second PIP joints.

JSW and cartilage thickness in the knee joints. The US-measured cartilage thickness for the lateral and medial femoral condyles correlated well with the radiography-measured JSW of the knees, with the strongest correlation for the medial condyle (Table 2).

The measurement of the JSW of the knee included the cartilage thickness of the femoral condyle and the tibia. For all evaluated measures, we found that the JSW as assessed by radiography was about $4 \mathrm{~mm}$ larger than the corresponding femoral cartilage thickness as measured by US. There was a significant intermodality correlation for all 4 anatomical spots: $\mathrm{r}=0.52-0.81$ and intraclass correlation coefficient $=0.458-0.721, \mathrm{p}<0.001$ (Table 2 and Figure 3). We found a tendency toward thinner cartilage and smaller JSW at the medial condyle compared to the lateral condyle except in the radiographic measures in the systemic JIA group and in the left knee of patients with persistent subtype oligoarthritis (subtype data not shown).

At all the spots at which the measurements were performed, we found that cartilage thickness or JSW decreased significantly with increasing age (data not shown). After adjusting for age and sex, we found thicker knee joint cartilage in patients with the oligoarticular subtype at the lateral, medial, and intercondylar spots. The smallest cartilage thickness values or JSW values were observed in the RF-positive polyarticular and systemic subtypes (data not shown).

In patients with a history of arthritis in the knees, the cartilage thickness as measured by US was smaller (statistically insignificant) than in knees without previous arthritis. However, when this was analyzed using radiographic JSW measures, there was a significant difference at the right medial condyle ( $\mathrm{p}=0.04$; Table 3 ).

$J S W$ and cartilage thickness in the finger joints. The US-measured cartilage thickness values for the finger joints

Personal non-commercial use only. The Journal of Rheumatology Copyright @ 2019 . All rights reserved. 

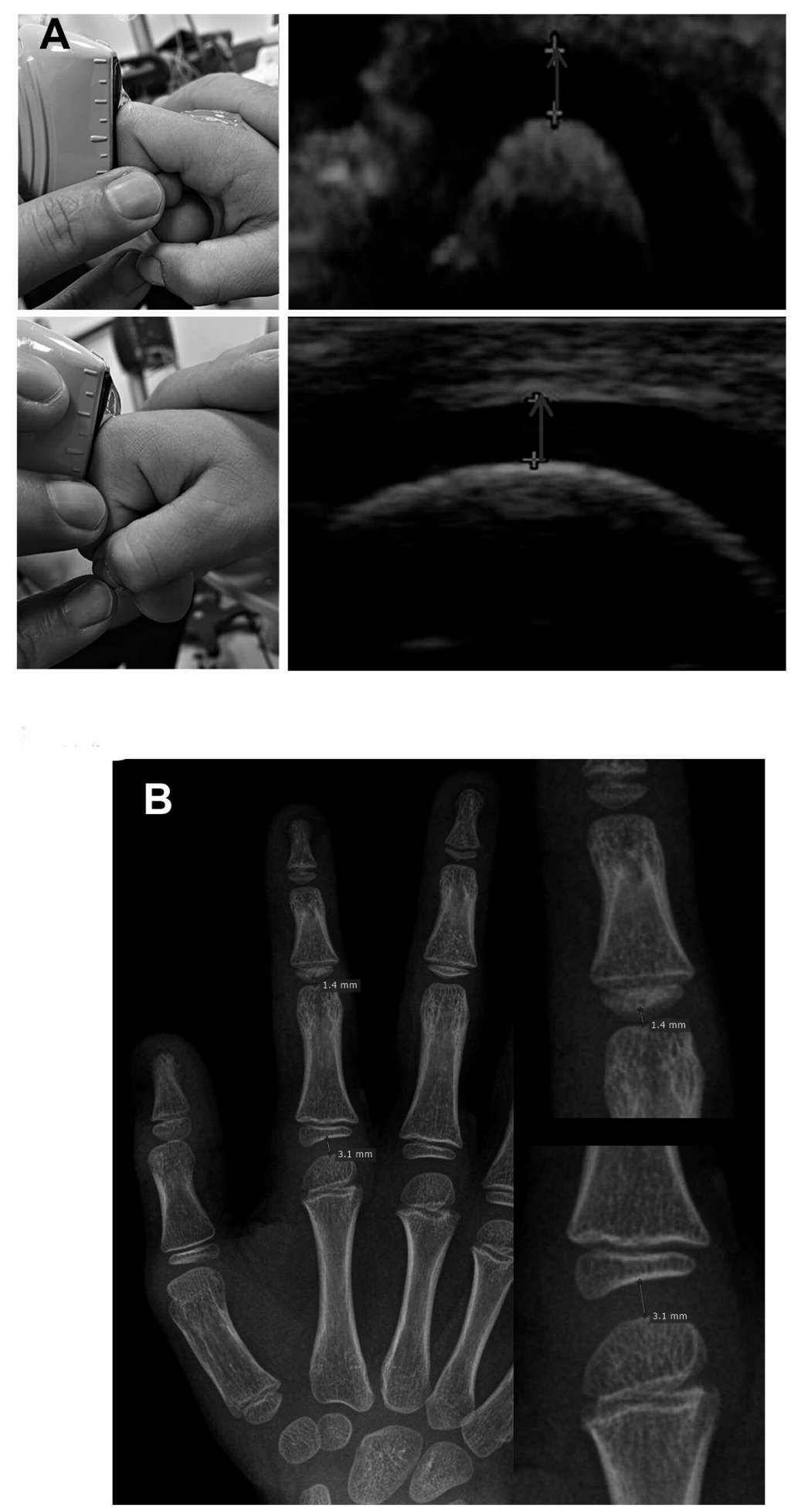

Figure 2. A. Ultrasound assessment of finger joint cartilage thickness. The grey arrows indicate the points at which the thickness was assessed. B. Radiograph assessment of finger joint space width (JSW). The grey line indicates where the JSW was assessed.

correlated well with the values of radiographically measured JSW, with the strongest correlation for the MCP joint (Table 2). The mean JSW was about $48 \%$ thicker than the US measurements for the MCP joint and $60 \%$ thicker for the PIP joint because the distal cartilage layer of the joints is included in the JSW measurement.

Personal non-commercial use only. The Journal of Rheumatology Copyright (C 2019. All rights reserved. 
Table 1. Demographic data of study population.

\begin{tabular}{|c|c|c|c|c|c|c|}
\hline Characteristics & $\begin{array}{c}\text { Oligo Persistent, } \\
\mathrm{n}=28\end{array}$ & $\begin{array}{l}\text { Oligo Extended, } \\
\quad \mathrm{n}=15\end{array}$ & $\begin{array}{l}\text { Poly RF-negative, } \\
\qquad \mathrm{n}=17\end{array}$ & $\begin{array}{l}\text { Poly RF-positive, } \\
\qquad \mathrm{n}=4\end{array}$ & $\begin{array}{l}\text { Systemic, } \\
\mathrm{n}=10\end{array}$ & $\begin{array}{c}\text { All, } \\
\mathrm{n}=74\end{array}$ \\
\hline Sex, girl (\%) & $20(71)$ & $12(80)$ & $15(88)$ & $4(100)$ & $3(30)$ & $54(73)$ \\
\hline Weight, kg, median (IQR) & $29.2(23.2-40.7)$ & $43.0(37.7-48.5)$ & $34.8(27.5-51.9)$ & $48.7(36.1-70)$ & $35.8(20.5-65.2)$ & $35.7(26.2-48.9)$ \\
\hline Height, cm, median (IQR) & $136.5(122-153)$ & $155(145-167)$ & $143(132-165)$ & $158(154-162)$ & $146(119-170)$ & $144(130-163)$ \\
\hline JADAS10, median (IQR) & $2.75(1-6.5)$ & $3(2-6)$ & $2(1-5.5)$ & $4.25(3.1-18.4)$ & $2(1.3-4.3)$ & $2.5(1-5)$ \\
\hline Disease duration, mos, median (IQR) & $36(16.3-59.5)$ & $83(60-134)$ & $51(31-98.5)$ & $21(9.8-36.5)$ & $55(29.8-74.3)$ & $44(28.8-86.3)$ \\
\hline \multicolumn{7}{|c|}{ History of active knee, $\mathrm{n}(\%$ of subtype) } \\
\hline Left & $17(61)$ & $11(73)$ & $12(71)$ & $4(100)$ & $3(30)$ & $47(64)$ \\
\hline Right & $18(64)$ & $12(80)$ & $15(88)$ & $4(100)$ & $6(60)$ & $55(74)$ \\
\hline \multicolumn{7}{|l|}{ IACI, $\mathrm{n}$ ( $\%$ of active knees) } \\
\hline Left & $12(71)$ & $10(91)$ & $11(92)$ & $4(100)$ & $2(67)$ & $39(83)$ \\
\hline Biologics & $1(4)$ & $6(40)$ & $7(41)$ & $1(25)$ & $1(10)$ & $16(22)$ \\
\hline
\end{tabular}

RF: rheumatoid factor; IQR: interquartile range; JADAS: Juvenile Arthritis Disease Activity Score; ANA: antinuclear antibodies; BMI: body mass index; IACI: intraarticular corticosteroid injection; NSAID: nonsteroidal antiinflammatory drug.

Table 2. Comparison of cartilage thickness measurements by US and JSW, assessed by radiography.

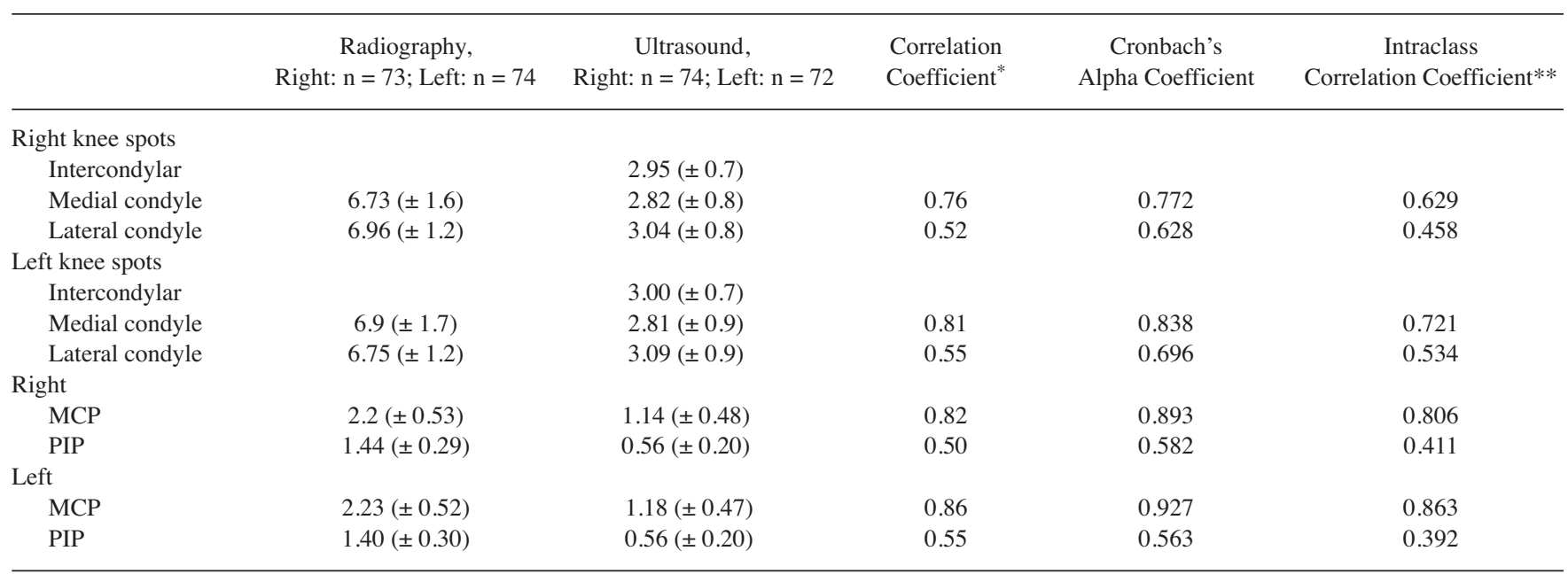

Cartilage thickness given as mm, mean $( \pm \mathrm{SD}) . *$ Spearman's rank: $\mathrm{p}<0.001$. ** Intraclass correlation coefficient: $\mathrm{p}<0.001$. US: ultrasound; JSW: joint space width; MCP: metacarpophalangeal; PIP: proximal interphalangeal.

There were no significant differences in the JSW or cartilage thickness between finger joints without or with previous/actual arthritis with either US or radiography. This may be because only the 21/136 MCP joints and the 18/138 PIP joints were affected (data not shown). We found a significant correlation between US and radiographic measures for both the MCP and PIP joints ( $\mathrm{r}=0.82-0.86$ and $0.50-0.55$, respectively, $\mathrm{p}<0.001$; Table 2, Figure 3 ). There were no significant differences in cartilage thickness or JSW according to JIA subcategory. However, we found significant decreases in cartilage thickness and JSW with increasing age in both the MCP and PIP joints.

\section{DISCUSSION}

The JSN in JIA is caused primarily by degeneration of the articular cartilage as a result of inflammatory activity in the synovium. The formation of pannus protrudes from the edges of the cartilage layer and causes the degradation. In our study, we found a close correlation between radiographic measures of JSW and cartilage thickness as assessed by US. In accordance with previous studies, we also found that patients with oligoarticular JIA had thicker knee cartilage than those with the polyarticular and systemic JIA subtypes ${ }^{9}$. In the same study we found a subtype difference for PIP joints but not for MCP joints. In the present study no difference was found in 

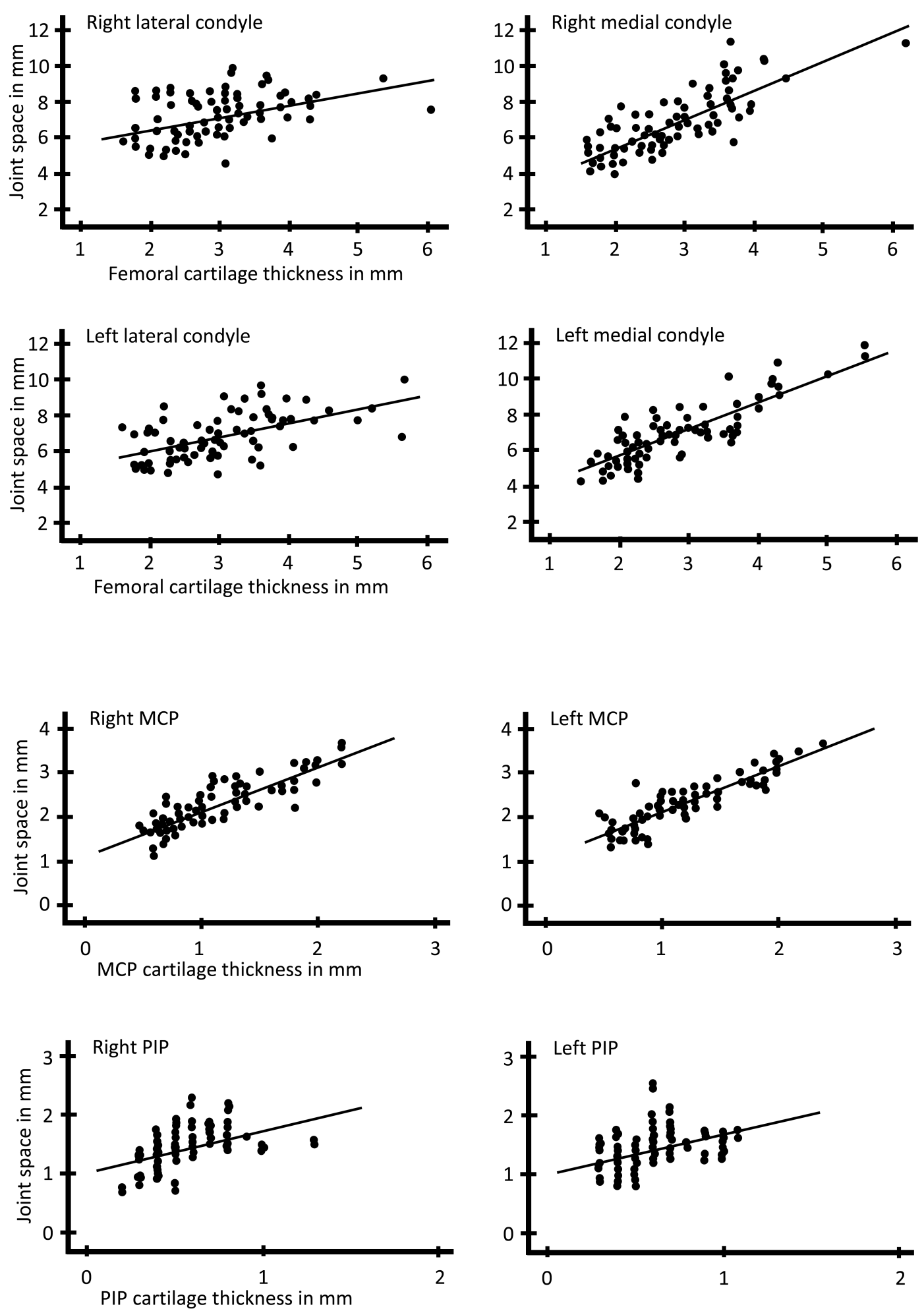

Figure 3. Scatter plots showing joint space width and cartilage thickness in the indicated joints where the width or thickness were assessed. MCP: metacarpophalangeal joint; PIP: proximal interphalangeal joint. 
Table 3. Comparison between knee joints with and without a previous history of clinical arthritic activity.

\begin{tabular}{|c|c|c|c|c|}
\hline & Active Knees & Not Active Knees & Estimated Difference* & $\mathrm{p}^{*}$ \\
\hline \multicolumn{5}{|l|}{ Right knee } \\
\hline US & $\mathrm{n}=55$ & $\mathrm{n}=19$ & & \\
\hline Medial & $2.7( \pm 0.7)$ & $3.13( \pm 1.1)$ & $0.15(-0.2$ to 0.5$)$ & 0.36 \\
\hline Radiography & $\mathrm{n}=55$ & $\mathrm{n}=18$ & & \\
\hline Lateral & $6.83( \pm 1.1)$ & $7.35( \pm 1.2)$ & $0.29(-0.4$ to 1.0$)$ & 0.41 \\
\hline US & $\mathrm{n}=46$ & $\mathrm{n}=26$ & & \\
\hline Lateral & $3.07( \pm 0.9)$ & $3.12( \pm 0.9)$ & $0.04(-0.3$ to 0.3$)$ & 0.79 \\
\hline Medial & $2.75( \pm 0.9)$ & $2.91( \pm 0.9)$ & $0.08(-0.2$ to 0.4$)$ & 0.55 \\
\hline Radiography & $\mathrm{n}=47$ & $\mathrm{n}=27$ & & \\
\hline Lateral & $6.57( \pm 1.1)$ & $7.07( \pm 1.2)$ & $0.40(-0.2$ to 1.0$)$ & 0.16 \\
\hline Medial & $6.64( \pm 1.6)$ & $7.34( \pm 1.9)$ & $0.47(-0.08$ to 1.0$)$ & 0.09 \\
\hline
\end{tabular}

Measurements in mm, mean $( \pm \mathrm{SD}) . *$ Regression analysis controlled for age, sex, and JIA subtype. US: ultrasound; JIA: juvenile idiopathic arthritis.

the finger joints according to subtype, which may be due to small sample sizes or a low percentage of finger joint involvement in the patients included. Because degradation of hyaline cartilage will not occur uniformly, the measures of joint cartilage thickness by US assessment should ideally be performed at multiple locations along the cartilage surface to accurately evaluate the cartilage damage. However, for comparison we have chosen the same setup as in our previous papers, using predefined scan planes for this study $6,7,8,9,10$. This was because the aim of our study was not to investigate the capability of radiography and US to show joint damage per se. Instead, we aimed to compare the 2 modalities in measuring JSW versus femoral cartilage thickness.

We found a close correlation between the radiographic and US measures. This may indicate that US assessment of articular cartilage thickness of only one of the articular bones could be used to diagnose JSN, thereby avoiding exposing the child to ionizing radiation. MRI was recently validated as a tool for assessing JSN in the hand and wrist joints in adults with rheumatoid arthritis ${ }^{13,14}$. MRI can be used to evaluate multiple joints in 1 session, making it possible to assess metacarpal joints where US cannot assess the cartilage orthogonally.

We found a high correlation between lateral and medial condyle measures, but there was also a tendency towards thinner cartilage at the medial spots compared to the lateral spots. This is in accordance with our previous US and MRI findings in a smaller cohort of patients with JIA ${ }^{10}$. Similarly, medial compartment disease is more prevalent than lateral compartment disease in patients with osteoarthritis in the $\mathrm{knee}^{15}$. However, there is a site difference in normal cartilage development, with lateral development being greater than medial development, and this may account for the difference seen in these studies ${ }^{16}$.

We demonstrated previously using US that there was decreased cartilage thickness in systemic and polyarticular
JIA compared to oligoarticular JIA ${ }^{9}$. In our present study, we found the same tendency for both US measures and for radiographically assessed JSW. Possibly as a result of the low number of patients with RF-positive polyarticular JIA in our cohort, we found no statistically significant difference compared to patients with oligoarticular JIA. The median cartilage thickness in the knee joints showed that patients in the polyarticular group had the thinnest cartilage among the JIA subtypes. The reason for this difference may be the disease course in children with polyarticular JIA, which often is more aggressive.

In our study, all US examinations were done by only 1 examiner without testing intrareader reliability, and radiograph measures of JSW were assessed by another examiner. Both investigators were blinded to each other's findings. We admit that the lack of testing for intrareader reliability may be a limitation of our study, although previously we have shown high interreader and intrareader reliability of US of selected joints in healthy children ${ }^{7}$.

Our results showed that the assessment of just the proximal cartilage site in both small and large joints correlated very well with the total measure of the JSW, including both proximal and distal cartilage sites. However, the conclusions of this study may not be generalized to cartilage in joints that are more difficult to access, such as the wrist and ankle joints. The measurement of JSW in the youngest children is difficult because of age-dependent ossification of the bones, resulting in wavy and fragmented irregularities of the bone surface, which may result in considerable variation between measurements.

We found that assessment of articular cartilage thickness by US in children with JIA was closely correlated to the joint space width measured radiographically. However, because our patient cohort had relatively few affected joints, further studies are needed (including patients with active arthritis) to conclude that US measurements of joint cartilage thickness

Personal non-commercial use only. The Journal of Rheumatology Copyright @ 2019 . All rights reserved. 
may be useful in prospective studies of JSN outcome measures of joint damage.

\section{REFERENCES}

1. Prakken B, Albani S, Martini A. Juvenile idiopathic arthritis. Lancet 2011;18:2138-49.

2. Sparling M, Malleson P, Wood B, Petty R. Radiographic followup of joints injected with triamcinolone hexacetonide for the management of childhood arthritis. Arthritis Rheum 1990;33:821-6.

3. Babyn PS. Pediatric musculoskeletal imaging. Semin Musculoskelet Radiol 2009;13:157.

4. van Rossum MA, Zwinderman AH, Boers M, Dijkmans BA, van Soesbergen RM, Fiselier TJ, et al. Radiologic features in juvenile idiopathic arthritis: a first step in the development of a standardized assessment method. Arthritis Rheum 2003;48:507-15.

5. Hufton AP, Doyle SM, Carty HM. Digital radiography in paediatrics: radiation dose considerations and magnitude of possible dose reduction. Br J Radiol 1998;71:186-99.

6. Spannow AH, Stenboeg E, Pfeiffer-Jensen M, Fiirgaard B, Haislund $\mathrm{M}$, Ostergaard M, et al. Ultrasound and MRI measurements of joint cartilage in healthy children: a validation study. Ultraschall Med 2011;32:110-6.

7. Spannow AH, Stenboeg E, Pfeiffer-Jensen M, Herlin T. Ultrasound measurement of joint cartilage thickness in large and small joints in healthy children: a clinical pilot study assessing observer variability. Pediatr Rheumatol Online J 2007;5:3.

8. Spannow AH, Pfeiffer-Jensen M, Andersen NT, Herlin T, Stenbog E. Ultrasonographic measurements of joint cartilage thickness in healthy children: age- and sex-related standard reference values. J Rheumatol 2010;37:2595-601.
9. Pradsgaard DO, Spannow AH, Heuck C, Herlin T. Decreased cartilage thickness in juvenile idiopathic arthritis assessed by ultrasonography. J Rheumatol 2013;40:1596-603.

10. Pradsgaard DO, Fiirgaard B, Spannow AH, Heuck C, Herlin T. Cartilage thickness of the knee joint in juvenile idiopathic arthritis: comparative assessment by ultrasonography and magnetic resonance imaging. J Rheumatol 2015;42:534-40.

11. Petty RE, Southwood TR, Manners P, Baum J, Glass DN, Goldenberg J, et al. International League of Associations for Rheumatology classification of juvenile idiopathic arthritis: second revision, Edmonton, 2001. J Rheumatol 2004;31:390-2.

12. Consolaro A, Ruperto N, Bazso A, Pistorio A, Magni-Manzoni S, Filocamo G, et al. Development and validation of a composite disease activity score for juvenile idiopathic arthritis. Arthritis Rheum 2009;61:658-66.

13. Ostergaard M, Boyesen P, Eshed I, Gandjbakhch F, Lillegraven S, Bird $\mathrm{P}$, et al. Development and preliminary validation of a magnetic resonance imaging joint space narrowing score for use in rheumatoid arthritis: potential adjunct to the OMERACT RA MRI scoring system. J Rheumatol 2011;38:2045-50.

14. Peterfy CG, Dicarlo JC, Olech E, Bagnard MA, Gabriele A, Gaylis $\mathrm{N}$. Evaluating joint-space narrowing and cartilage loss in rheumatoid arthritis by using MRI. Arthritis Res Ther 2012;14:R131

15. Ledingham J, Regan M, Jones A, Doherty M. Radiographic patterns and associations of osteoarthritis of the knee in patients referred to hospital. Ann Rheum Dis 1993;52:520-6.

16. Jones G, Glisson M, Hynes K, Cicuttini F. Sex and site differences in cartilage development: a possible explanation for variations in knee osteoarthritis in later life. Arthritis Rheum 2000;43:2543-9. 